\title{
Particle-in-cell simulations of return current in solar flares
}

\author{
M. Karlický, D. H. Nickeler, and M. Bárta \\ Astronomical Institute of the Academy of Sciences of the Czech Republic, 25165 Ondřejov, Czech Republic \\ e-mail: [karlicky;nickeler; barta]@asu.cas.cz
}

Received 25 January 2008 / Accepted 18 April 2008

\begin{abstract}
Aims. We numerically study a formation of the return current generated in solar flares.

Methods. For simulations of the return current in the beam-plasma system, a 3D particle-in-cell electromagnetic code is used. Results. In conditions of solar flares with the electron beam fluxes of $E_{\mathrm{F}}=9.1 \times 10^{9}-4.55 \times 10^{10} \mathrm{erg} \mathrm{s}^{-1} \mathrm{~cm}^{-2}$, the beam-plasma interaction with the return current is studied. We found that the electron beam relaxes to the plateau distribution function as known from electrostatic simulations. Simultaneously, due to electromagnetic effects and the Buneman instability of the prescribed Maxwellshifted return current, the electron distribution function evolves to a new stationary state with a new form of the return current. In this final state the return current is formed not only by electrons in the bulk of the electron distribution function, but also by electrons in the extended tail. We use the results of simulations to estimate the critical beam fluxes for the processes under study in the low corona, the transition region and the upper chromosphere.
\end{abstract}

Key words. Sun: flares - Sun: chromosphere - Sun: transition region

\section{Introduction}

Electron beams accelerated in solar flares bombard deep layers of the solar atmosphere. They propagate from the flare acceleration site through the low corona and transition region to the chromosphere where the beams are decelerated and produce hard $\mathrm{X}$-ray emissions. These electron beams with the estimated fluxes of $E_{\mathrm{F}}=10^{9}-10^{12} \mathrm{erg} \mathrm{s}^{-1} \mathrm{~cm}^{-2}$ represent huge electric currents that have to be neutralized by the return currents (Hoyng et al. 1978; van den Oord 1990). Usually it is assumed that the return current is formed by all background plasma electrons having the same drift velocity - the Maxwell electron distribution shifted in the velocity space compared to the protons (e.g. Karlický \& Hénoux 1992). On the other hand, Rowland \& Vlahos (1985) proposed that the return current is formed by so-called runaway electrons for such huge fluxes. Their idea essentially reduces the return current electric field and thus decreases the energy losses of the electron beam penetrating the chromosphere. From a physical point of view, formation of the return current has two aspects: a) electric charge effects at the front of the electron beam and $b$ ) electromagnetic effects of the beam electric current. For the details, see the paper by van den Oord (1990).

Effects of the return current on the dynamics of nonthermal electron beams and the heating of the flare atmosphere have been described and studied by Emslie (1980), Karlický et al. (1990), Karlický \& Hénoux (1992), and Zharkova et al. (1995).

The papers (Karlický \& Hénoux 2002; Hénoux \& Karlický 2003; Karlický et al. 2004; Štepán et al. 2007) proposed that the polarization and enhanced intensities of the chromospheric optical lines can be explained by the formation of the return current at chromospheric layers. It was found that the resulting intensities and polarization are very sensitive to the form of the electron distribution function (with the return current), especially in the range of the excitation and ionization energies of these lines.
Similarly, the return current can modify the electron distribution function in the low corona and thus influence the intensities of the EUV and soft X-ray lines observed during solar flares (Dzifčáková \& Mason 2008).

Thus, a knowledge of the detailed form of the electron distribution function in the whole flare atmosphere during the electron beam propagation is very important. In this paper, we want to contribute to a solution of this complex problem. Using the 3D particle-in-cell (PIC) electromagnetic code, we study an evolution of a beam in the plasma with the return current.

\section{Model}

To simulate the formation of the return current, we used a 3D (3 spatial and 3 velocity components), relativistic electromagnetic PIC code (Buneman 1993). The system size was chosen as $L_{x}=10 \Delta, L_{y}=10 \Delta$, and $L_{z}=805 \Delta$, where $\Delta$ is the grid size. The short dimensions in $x$ and $y$ coordinates are given by the computer limits.

In the model we initiated a spatially homogenous electronproton plasma with the proton-electron mass ratio $m_{\mathrm{p}} / m_{\mathrm{e}}=16$ or 100 (considered for better description of the dynamics of protons) and temperatures $T_{\mathrm{e}}=T_{\mathrm{i}}=1 \times 10^{6}-2 \times 10^{6} \mathrm{~K}$ (the temperature corresponding to the low corona). Four hundred electrons and four hundred protons per the cube grid were taken. The plasma frequency is $\omega_{\mathrm{pe}}=0.01$, and the electron Debye length is $\lambda_{\mathrm{D}}=0.65 \Delta$.

Furthermore, the electron beam that is homogenous in space with the velocity in the $z$ direction $v_{\mathrm{b}} / c=1 / 3$ (20 electrons per the grid cube) is included, and $c$ is the speed of light. To keep the charge neutrality, the same amount of protons with zero velocities are added. (Note: such an initiation of the electron beam means that only electromagnetic aspects of the return current 


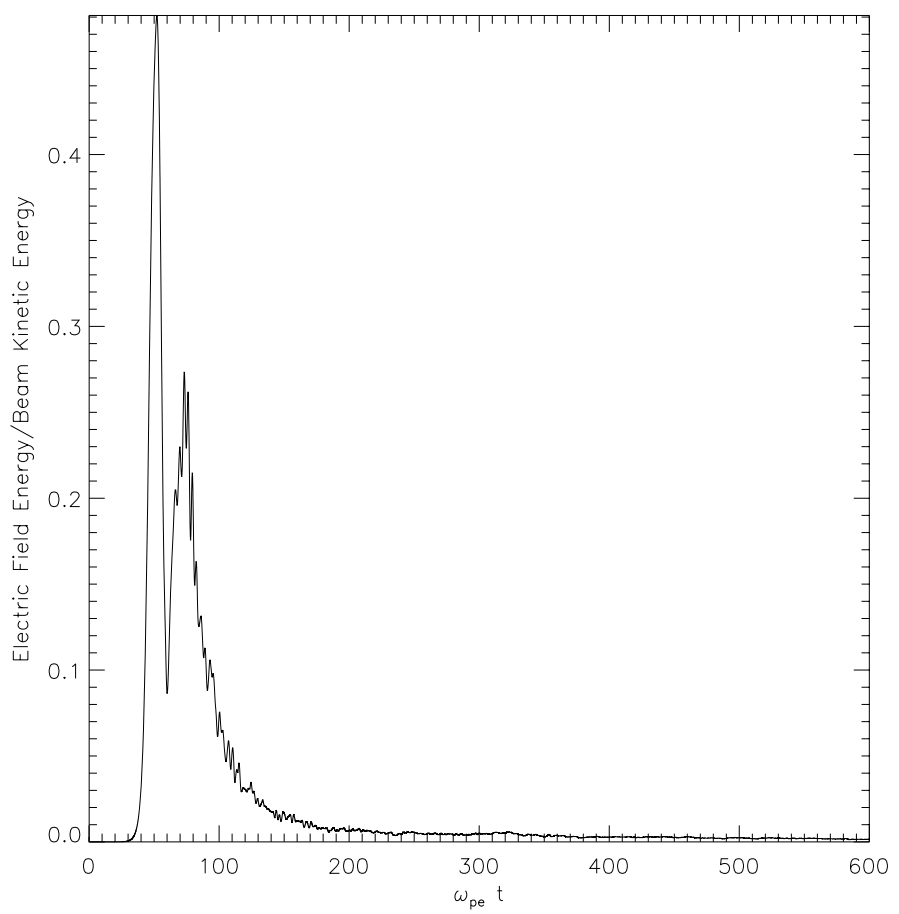

Fig. 1. The time evolution of the electric field energy (Case A).

formation were studied. The electrostatic effects at the beam front were not taken into account.) The ratio of the beam and plasma densities were chosen $n_{\mathrm{b}} / n_{\mathrm{e}}=0.1,0.05$, and 0.02 . Considering the density in the low corona as $n_{\mathrm{e}}=10^{9} \mathrm{~cm}^{-3}$, the corresponding beam densities are $n_{\mathrm{b}}=0.2 \times 10^{8}-1.0 \times$ $10^{8} \mathrm{~cm}^{-3}$, i.e. the beam energy flux is $E_{\mathrm{F}}=9.1 \times 10^{9}-4.55 \times$ $10^{10} \mathrm{erg} \mathrm{s}^{-1} \mathrm{~cm}^{-2}$, which is in the range of expected fluxes.

To neutralize the beam's electric current, in the initial state we prescribed the return current by adding the same drift velocity to all background plasma electrons (we shifted the Maxwell electron distribution in the velocity space compared to the protons) according to the relation $v_{\mathrm{D}}=-v_{\mathrm{b}} n_{\mathrm{b}} / n_{\mathrm{e}}$. For this type of initiation see also Niemiec et al. (2008). The magnetic field in the initial state is taken as zero, and the periodic boundary conditions are used.

All computations were performed on the parallel computer OCAS (Ondřejov Cluster for Astrophysical Simulations), see http: //wave.asu.cas.cz/ocas.

\section{Results of simulations}

First, the results of simulations relevant to the low corona with the beam of the energy flux $E_{\mathrm{F}}=2.27 \times 10^{10} \mathrm{erg} \mathrm{s}^{-1} \mathrm{~cm}^{-2}$ and $n_{\mathrm{b}} / n_{\mathrm{e}}=1 / 20, T_{\mathrm{e}}=T_{\mathrm{i}}=10^{6} \mathrm{~K}, m_{\mathrm{i}} / m_{\mathrm{e}}=16$ are presented (Case A). Figure 1 shows an evolution of the electric field energy that is dominant in its z-component, i.e. in the direction of electron beam propagation. The energy starts from the noise level, then exponentially increases, and it decreases after its saturation at $\omega_{\mathrm{pe}} t=50$. The exponential growth rate $\gamma$ and the energy saturation level $P E$ correspond approximately to the analytical relations derived for the cold beam - plasma instability (Michailovskij 1975; Drummond et al. 1970):

$\gamma=\omega_{\mathrm{pe}} \sqrt{3} \alpha^{1 / 3} / 2^{4 / 3}$,

$P E=K E_{\text {beam }}(\alpha / 2)^{1 / 3}$,

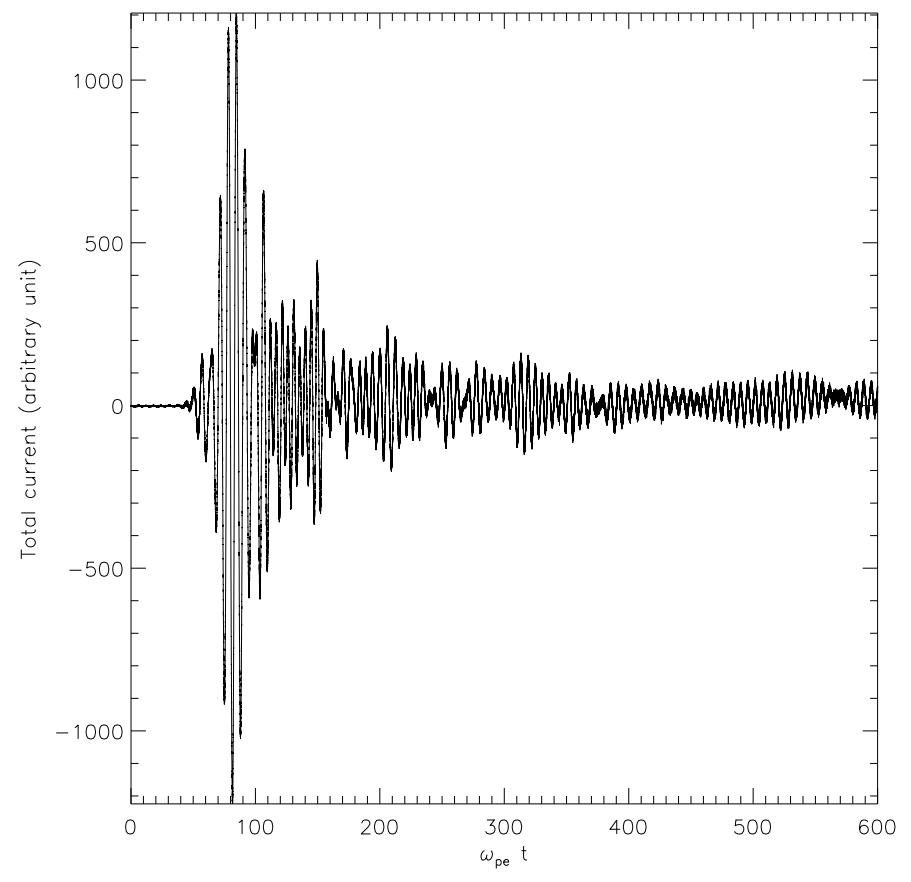

Fig. 2. The time evolution of the total electric current averaged over the whole volume of the system (Case A).

where $\alpha$ is the ratio of the beam and plasma densities, and $K E_{\text {beam }}$ the kinetic beam energy.

On the other hand, in the initial state of this case, we prescribed the return current by a velocity shift of the distribution function of the background plasma electrons compared to protons as $v_{\mathrm{D}}=-v_{\mathrm{b}} / 20=-1.285 v_{\mathrm{Te}}$, where $v_{\mathrm{Te}}$ is the thermal velocity of plasma electrons. Thus, the return current is in the regime of the Buneman instability (Michailovskij 1975). Both the beamplasma and Buneman instabilities produce plasma waves that influence the total current in the system (see the strong variations in the total current at $\omega_{\mathrm{pe}} t=50-100$ in Fig. 2) and modify the whole electron distribution function such as a formation of the plateau on the beam side of the distribution function and the return current on the opposite side in the stationary state at $\omega_{\text {pe }} t=$ 600 in Fig. 3. (Note: the total current in Fig. 2 means the electric current is averaged over the whole volume of the system.) The detailed view of the same distribution function for a lower velocity range is presented in Fig. 4. From both Figs. 3, and 4 we can see that the return current is formed not only by electrons in the bulk of the distribution function, but also by electrons in the extended tail. The distribution functions in velocities perpendicular to the beam direction $\left(v_{x}\right.$ and $\left.v_{y}\right)$ are only weakly influenced by these processes (Figs. 3 and 4). For the purpose of the spectroscopy of the low corona, we added the energy distribution functions corresponding to the initial and final states in Fig. 5.

Now for comparison, in Figs. 6 and 7 we present the results of further simulations: a) Case B, all parameters are the same as in the case $\mathrm{A}$, except that the proton-electron mass ratio is $m_{\mathrm{i}} / m_{\mathrm{e}}=100$, and $\mathrm{b}$ ) the case $\mathrm{C}$ in which we chose the same parameters as in case $\mathrm{A}$, except that the proton-electron mass ratio is $m_{\mathrm{i}} / m_{\mathrm{e}}=100$, and the ratio of the beam and plasma densities is $n_{\mathrm{b}} / n_{\mathrm{e}}=0.1$. The energy beam flux in Case $\mathrm{C}$ is $E_{\mathrm{F}}=$ $4.55 \times 10^{10} \mathrm{erg} \mathrm{s}^{-1} \mathrm{~cm}^{-2}$. Comparing all these presented cases, we found that the extent of the tail of the distribution function on the return current side increases with the increase in the parameter $n_{\mathrm{b}} / n_{\mathrm{e}}$ and with the decrease in the proton-electron mass ratio (see Fig. 8). Furthermore, the additional computations where we 

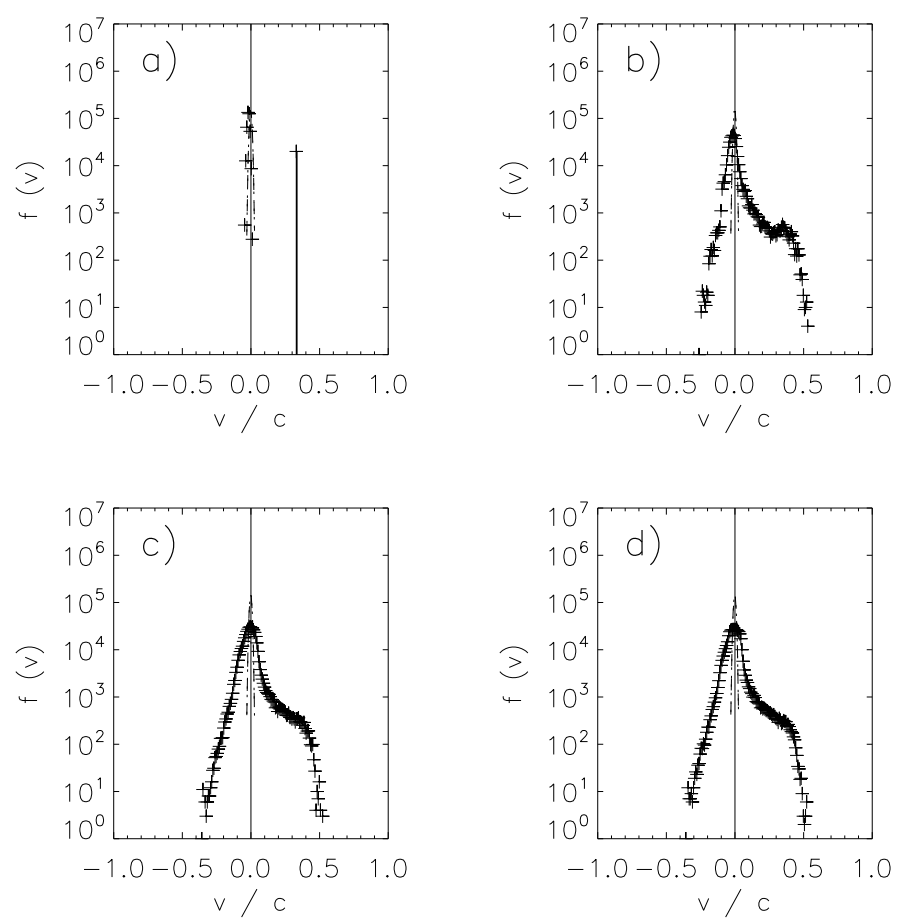

Fig. 3. The electron distribution functions (the crosses mean $f\left(v_{z}\right)$, dotdashed line means $f\left(v_{x}\right)$ or $\left.f\left(v_{y}\right)\right)$ at four different times: at the initial state a), at $\left.\omega_{\mathrm{pe}} t=100 \mathbf{b}\right)$, at $\omega_{\mathrm{pe}} t=300 \mathbf{c}$ ), and $\left.\omega_{\mathrm{pe}} t=600 \mathbf{d}\right)$ (Case A).
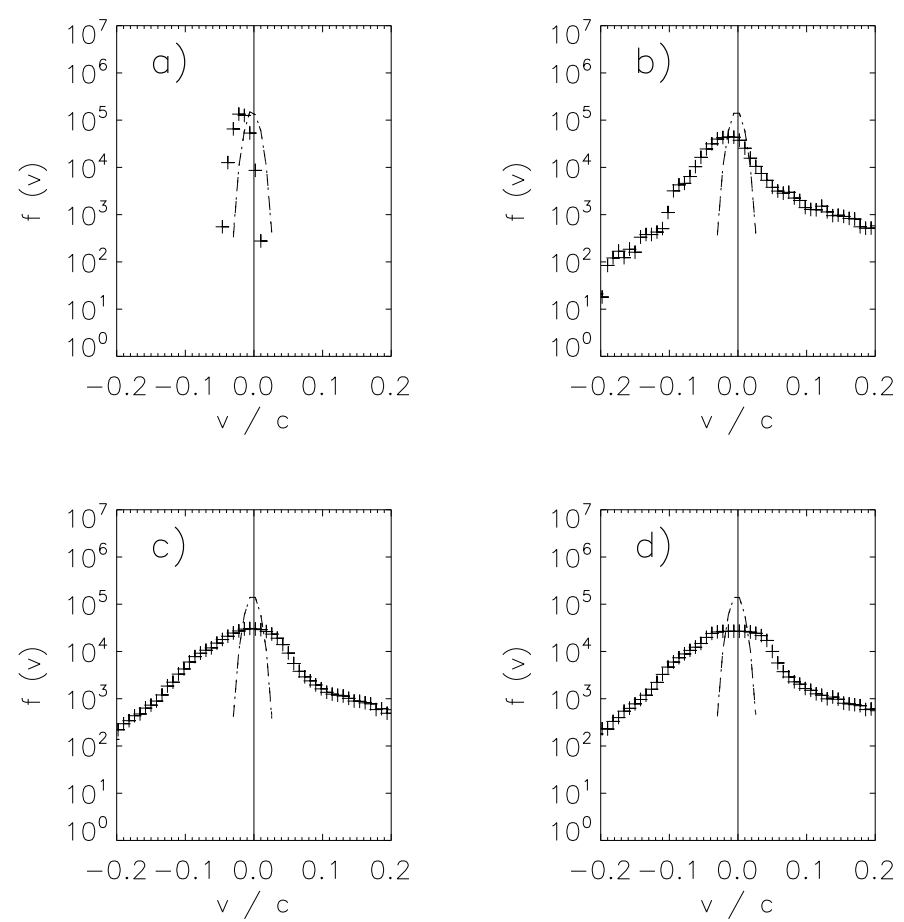

Fig. 4. Detailed view on the bulk of the same distribution functions as in Fig. 3.

changed the plasma temperature reveal that this extent of the return current tail decreases with the increase in the plasma temperature. For example, for $T_{\mathrm{e}}=T_{\mathrm{i}}=20 \times 10^{6} \mathrm{~K}$, and $n_{\mathrm{b}} / n_{\mathrm{e}}=0.1$ the initial and the final forms of the return current are the same; i.e., no modification of the initial prescribed return current was found.

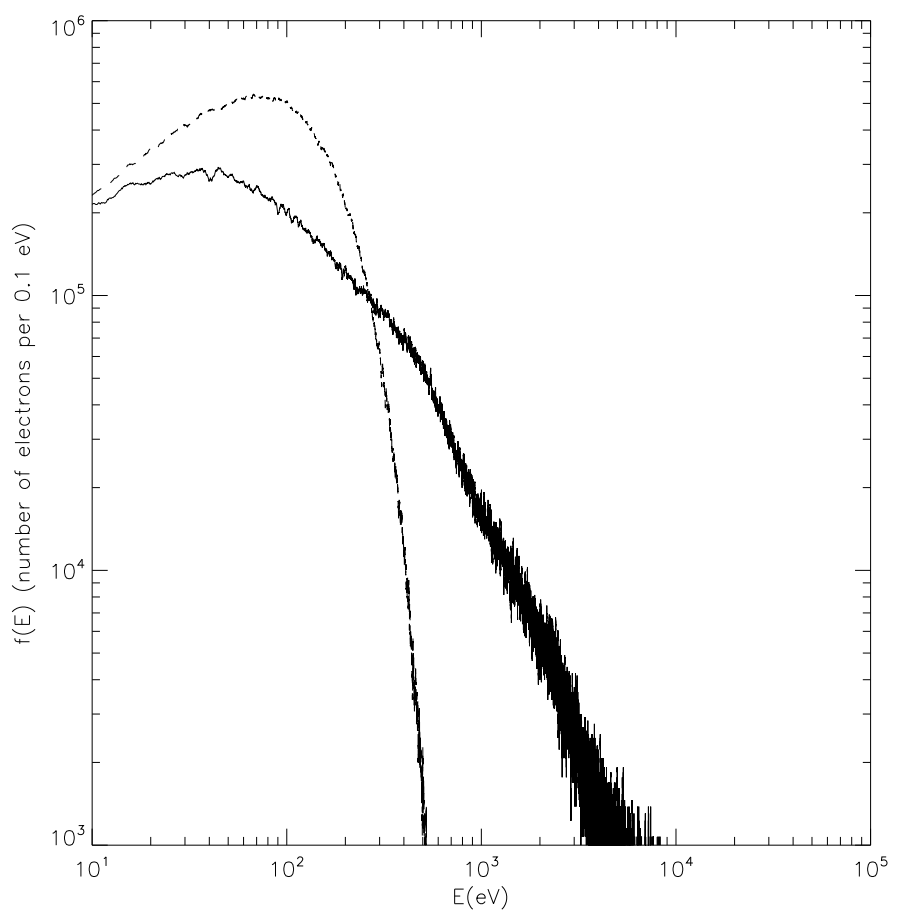

Fig. 5. The energy electron distribution function of the plasma in the low corona with the density $n_{\mathrm{e}}=10^{9} \mathrm{~cm}^{-3}$ and the temperature $T_{\mathrm{e}}=$ $10^{6} \mathrm{~K}$ (dashed line). The same distribution function at $\omega_{\mathrm{pe}} t=600$, i.e. after the quasi-linear relaxation of the electron beam and return-current formation processes (full line, Case A).
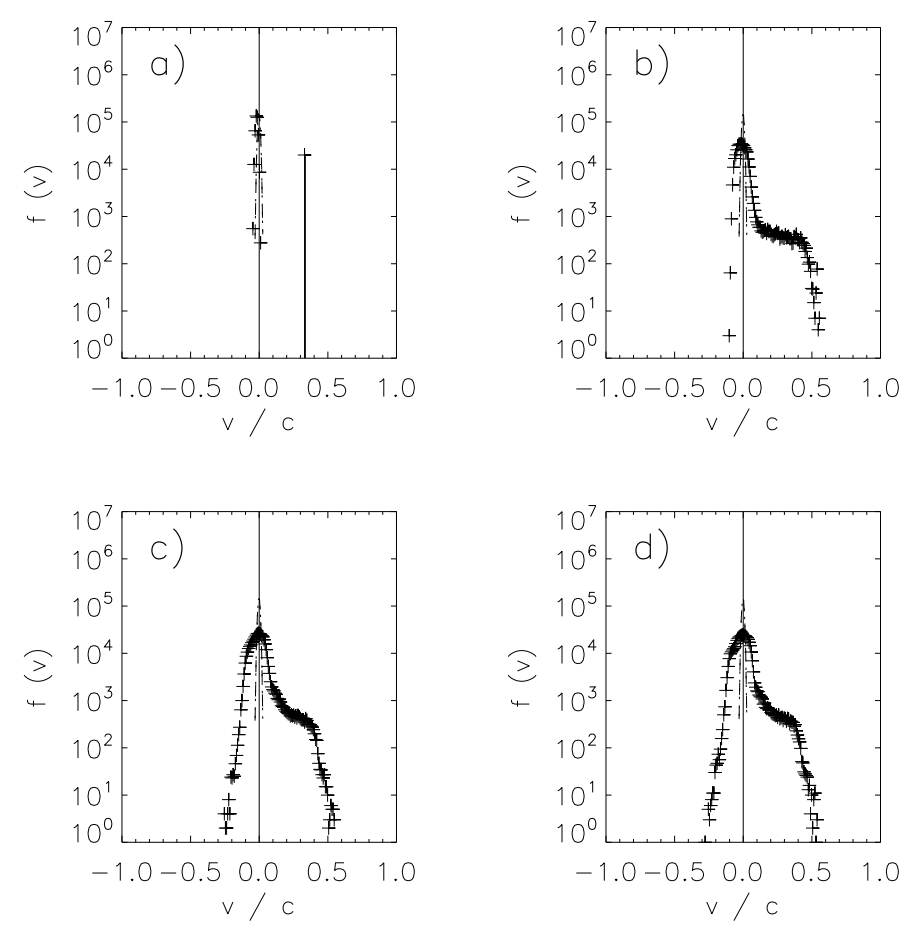

Fig. 6. The electron distribution functions (the crosses mean $f\left(v_{z}\right)$, dotdashed line means $f\left(v_{x}\right)$ or $\left.f\left(v_{y}\right)\right)$ at four different times: at the initial state a), at $\left.\omega_{\mathrm{pe}} t=100 \mathbf{b}\right)$, at $\left.\omega_{\mathrm{pe}} t=300 \mathbf{c}\right)$, and $\left.\omega_{\mathrm{pe}} t=600 \mathbf{d}\right)($ Case B).

\section{Discussion and conclusions}

Using a 3D particle-in-cell model, we studied the evolution of the return current. We found that the electron beam relaxes to the plateau distribution function. Simultaneously, the Langmuir 

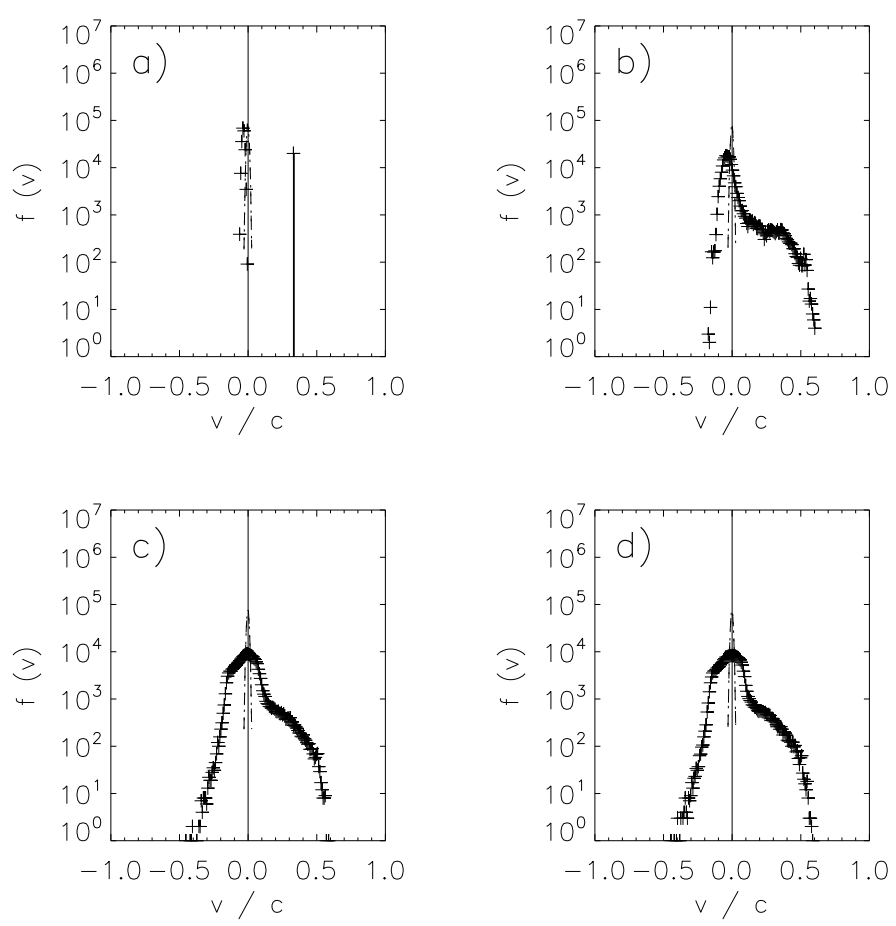

Fig. 7. The electron distribution functions (the crosses mean $f\left(v_{z}\right)$, dotdashed line means $f\left(v_{x}\right)$ or $\left.f\left(v_{y}\right)\right)$ at four different times: at the initial state a), at $\left.\omega_{\mathrm{pe}} t=100 \mathbf{b}\right)$, at $\omega_{\mathrm{pe}} t=300 \mathbf{c}$ ), and $\left.\omega_{\mathrm{pe}} t=600 \mathbf{d}\right)$ (Case C).

waves are generated, as is known from electrostatic simulations (e.g. Grognard 1985).

But due to a consideration of electromagnetic effects and due to in all these cases the initial return current is in the Buneman instability regime $\left(v_{\mathrm{D}}>v_{\mathrm{Te}}\right)$, the return current evolves into a new stationary state with the extended tail of the electron distribution function on the return current side. Also, the distribution function on the beam side close to the bulk is enhanced.

The resulting return current is formed not only by electrons in the bulk of the electron distribution function, but also by electrons from its extended tail. It reconciles the model of the return current formed by all electrons having the same drift velocity (e.g. Karlický \& Hénoux 1992) with the model explaining the return current by the runaway electrons alone (Rowland \& Vlahos 1985).

The results of simulations show that the extent of the superthermal tail of the distribution function on the return current side increases with the increase in the parameter $n_{\mathrm{b}} / n_{\mathrm{e}}$ and with the decrease in the proton-electron mass ratio $m_{\mathrm{i}} / m_{\mathrm{e}}$ and the electron plasma temperature $T_{\mathrm{e}}$.

These dependencies can be explained by the threshold and the growth rate of the Buneman instability. Namely, the higher value of $n_{\mathrm{b}} / n_{\mathrm{e}}$ corresponds to the higher value of $v_{\mathrm{D}} / v_{\mathrm{Te}}$, and the instability growth rate is proportional to $\left(m_{\mathrm{e}} / m_{\mathrm{i}}\right)^{1 / 2}$ (Michailovskij 1975; Ramirez-Ruiz et al. 2007).

Because the processes leading to the redistribution of the return current from the shifted Maxwellian distribution function to the one with the extended electron distribution tail depend on the threshold for the Buneman instability, we can estimate the critical beam fluxes in the whole flare atmosphere from the low corona to the chromosphere. Putting the Buneman instability threshold as $v_{\mathrm{D}}>v_{\mathrm{Te}}$, the critical particle beam flux $F_{n}$ can be expressed as

$F_{n}=n_{\mathrm{e}} v_{\mathrm{Te}}$.

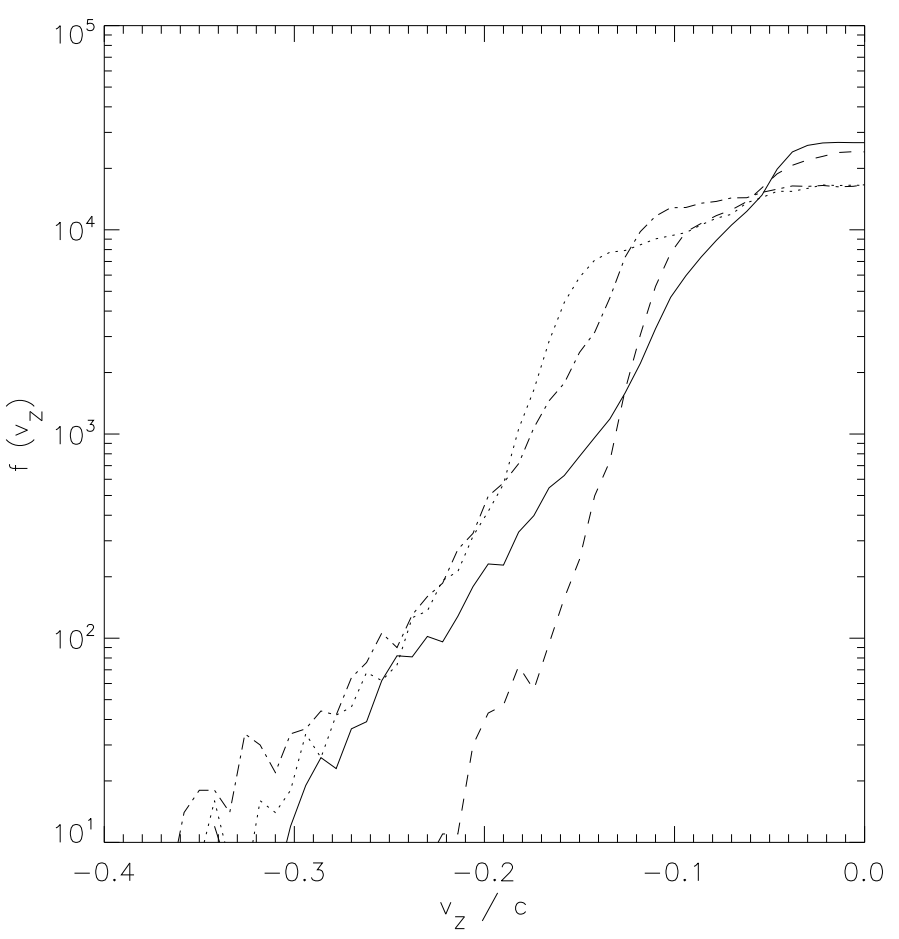

Fig. 8. Comparison of the extent of the superthermal tail of the distribution functions on the return current side at $\omega_{\text {pe }} t=600$. The full line corresponds to Case A $\left(n_{\mathrm{b}} / n_{\mathrm{e}}=0.05, m_{\mathrm{i}} / m_{\mathrm{e}}=16\right)$, the dashed line to Case B $\left(n_{\mathrm{b}} / n_{\mathrm{e}}=0.05, m_{\mathrm{i}} / m_{\mathrm{e}}=100\right)$, dotted line to Case C $\left(n_{\mathrm{b}} / n_{\mathrm{e}}=0.1\right.$, $\left.m_{\mathrm{i}} / m_{\mathrm{e}}=100\right)$, and dot-dashed line to the same case as Case $\mathrm{C}$, but with the proton-electron mass ratio $m_{\mathrm{i}} / m_{\mathrm{e}}=16$.

Table 1. Critical particle and energy beam fluxes.

\begin{tabular}{cccc}
\hline \hline $\begin{array}{c}\text { Atmospheric } \\
\text { layer }\end{array}$ & $\begin{array}{c}\text { Low } \\
\text { corona }\end{array}$ & $\begin{array}{c}\text { Transition } \\
\text { region }\end{array}$ & $\begin{array}{c}\text { Upper } \\
\text { chromosphere }\end{array}$ \\
\hline$n_{\mathrm{e}}{ }^{a}$ & $10^{9}$ & $10^{10}$ & $10^{12}$ \\
$T_{\mathrm{e}}{ }^{b}$ & $10^{6}$ & $10^{5}$ & $10^{4}$ \\
$F_{n c}{ }^{c}$ & $3.89 \times 10^{17}$ & $1.23 \times 10^{18}$ & $3.89 \times 10^{19}$ \\
$F_{e c}{ }^{d}$ & $1.77 \times 10^{10}$ & $5.56 \times 10^{10}$ & $1.77 \times 10^{12}$ \\
\hline
\end{tabular}

${ }^{a}$ Electron density $\left(\mathrm{cm}^{-3}\right) ;{ }^{b}$ temperature $(\mathrm{K}) ;{ }^{c}$ critical particle flux $\left(\mathrm{s}^{-1} \mathrm{~cm}^{-2}\right) ;{ }^{d}$ critical energy flux ( $\left.\mathrm{erg} \mathrm{s}^{-1} \mathrm{~cm}^{-2}\right)$.

This means that, if the particle beam flux is greater than this critical flux, then the extended tail on the return-current side of the electron distribution function is formed. Otherwise, the return current will have the form of the shifted Maxwell distribution. The critical particle beam fluxes, together with the critical energy beam fluxes in the low corona, transition region, and the upper chromosphere are presented in Table 1. (The critical energy beam fluxes were computed under an assumption of the monoenergetic beam with the velocity $v_{\mathrm{b}}=10^{10} \mathrm{~cm} \mathrm{~s}^{-1}$.) It shows that for sufficiently high (but still realistic) beam fluxes, the processes under study can lead to the extended tail of the distribution function on the return current side in all layers of the solar atmosphere and thus to influence on the emissions from these layers. The presented Cases A-C correspond to the supercritical regime (i.e. in the Buneman instability regime) in the low corona.

Due to limits of our computer we considered the numerical box with short dimensions in the $x$ and $y$ coordinates, i.e. in the perpendicular directions to the electron beam propagation. Because in this case the ion skin depth $\left(\lambda_{\mathrm{ci}}=c / \omega_{\mathrm{pi}}\right.$, where 
$\omega_{\text {pi }}$ is the ion plasma frequency) is greater than these perpendicular dimensions, even for the low proton-electron mass ratios (proportional to $\left.\left(m_{\mathrm{i}} / m_{\mathrm{e}}\right)^{1 / 2}\right)$, the oblique wave modes are not excited properly in this system. It limits all our results. We plan to extend these perpendicular dimensions because there are other important processes that can influence the resulting form of the return current. The most important is the Weibel instability (Weibel 1959), which is the electromagnetic instability in contrast to the electrostatic bump-on tail and Buneman instabilities. As shown by Medvedev \& Loeb (1999), Bret et al. (2005), Bret (2006), Rowlands et al. (2007), and Dieckmann et al. (2007), the Weibel instability can generate current filaments and associated magnetic fields in such plasma systems. These processes more accelerate particles (Nishikawa et al. 2006, 2007, 2008) and thus more can influence the return current formation, meaning that in future work all these processes need to be taken into account.

Acknowledgements. This research was supported by the Center for Theoretical Astrophysics, Prague and by Grant IAA300030701 of the Grant Agency of the Academy of Sciences of the Czech Republic. The authors thank the referee, Dr. Ken-Ichi Nishikawa, for constructive comments that essentially improved the paper.

\section{References}

Bret, A. 2006, Europhys. Lett., 74, 1027

Bret, A., Firpo, M.-C., \& Deutsch, C. 2005, Phys. Rev. Lett., 94 (11), 115002

Buneman, O. 1993, in Computer Space Plasma Physics, ed. H. Matsumoto, \& Y. Omura (Tokyo: Terra Scientific Publ. Comp.), 305
Dieckmann, M. E., Lerche, I., Shukla, P. K., \& Drury, L. O. C. 2007, New J. Phys., 9, 10

Drummond, W. E., Malberg, J. H., O’Neil, T. M., \& Thompson, J. R. 1970, Phys. Fluids, 13, 2422

Dzifč́áková, E., \& Mason, H. 2008, Sol. Phys., 247, 301

Emslie, A. G. 1980, ApJ, 235, 1055

Grognard, R. J. 1985, in Solar Radiophysics, ed. D. J. McLean, \& N. R. Labrum (Cambridge, USA: Cambridge University Press), 253

Hénoux, J. C., \& Karlický, M. 2003, A\&A, 407, 1103

Hoyng, P., Knight, J. W., \& Spicer, D. S. 1978, Solar Phys., 58, 139

Karlický, M., \& Hénoux, J. C. 1992, A\&A, 264, 679

Karlický, M., \& Hénoux, J. C. 2002, A\&A, 383, 713

Karlický, M., Alexander, D., Brown, J. C., \& MacKinnon, A. L. 1990, Solar Phys., 129, 325

Karlický, M., Kašparová, J., \& Heinzel, P. 2004, A\&A, 416, L13

Medvedev, M. V., \& Loeb, A. 1999, ApJ, 526, 697

Michailovskij, A. B. 1975, Teorija plazmennych neustojchivostej, Atomizdat, Moskva (in Russian)

Niemiec, J., Pohl, M., Stroman, T., \& Nishikawa, K. 2008, ApJ, submitted [arXiv:0802.2185]

Nishikawa, K.-I., Hardee, P. E., Hededal, C. B., \& Fishman, G. J. 2006, ApJ, 642,1267

Nishikawa, K.-I., Hededal, C. B., Hardee, P. E., et al. 2007, Ap\&SS, 307, 319

Nishikawa, K.-I., Hardee, P., Mizuno, Y., et al. 2008, Proc. of the Seventh European Workshop on Collisionless Shocks, Paris 2007 [arXiv: 0802.2558]

Ramirez-Ruiz, E., Nishikawa, K.-I., \& Hededal, Ch. B. 2007, ApJ, 671, 1877

Rowland, H. L., \& Vlahos, L. 1985, A\&A, 142, 219

Rowlands, G., Dieckmann, M. E., \& Shukla, P. K. 2007, New J. Phys., 9(8), 247

Štepán, J. A., Kašparová, J., Karlický, M., \& Heinzel, P. 2007, A\&A, 472, 55

van den Oord, G. H. 1990, A\&A, 234, 496

Weibel, E. S. 1959, Phys. Rev. Lett., 2, 83

Zharkova, V. V., Brown, J. C., \& Syniavskii, D. V. 1995, A\&A, 304, 284 